\title{
A Failure of Heart in Rheumatoid Arthritis
}

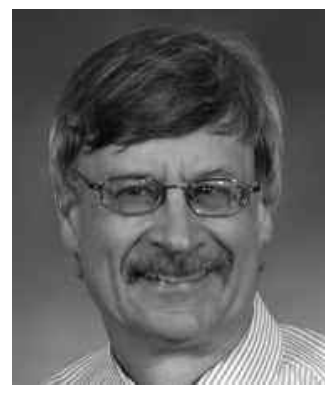

Rheumatoid arthritis (RA) is a systemic disease we treat with medications that have systemic effects. It is becoming increasingly clear that patients with RA have accelerated atherosclerosis and that RA is an independent risk factor for the development of atherosclerotic coronary artery disease ${ }^{1}$. What is less clear is whether the presence of RA and its therapies modulates the course of coronary artery disease and its complications. In this issue of The Journal Myasoedova, et al use their extensive database from the Mayo Clinic to explore heart failure in patients with $\mathrm{RA}^{2}$.

The relationship between heart failure and RA is not clear-cut. Some studies suggest that heart failure may be more frequent in patients with $\mathrm{RA}^{3}$, whereas other studies suggest the opposite ${ }^{4}$. Some of these differences can be explained by the more specific questions that the investigators are asking. Moreover, most studies of this problem are observational and depend on clinical diagnoses made during the course of clinical management, like the one in this issue. Thus, the presence or absence of heart failure is not systematically examined with questionnaires or echocardiograms, for example. This can be problematic in our effort to understand heart failure in RA patients since patients and physicians may ascribe symptoms such as ankle swelling as well as functional limitations to RA that may instead be a consequence of heart failure. Interestingly, studies that systemically used echocardiograms found that compared to other patients, patients with RA more likely had preserved ejection fractions, suggesting greater problems with diastolic rather than systolic function compared to patients without $\mathrm{RA}^{5}$. Moreover, while it is tempting to ascribe heart failure to ischemic heart disease, other studies suggest that in RA patients, factors other than ischemic heart disease and its risk factors more likely contribute to heart failure ${ }^{6}$.

The current study and others suggest a prime role for inflammation in the development of heart failure in RA, whether it more directly affects cardiac muscle or through enhanced atherosclerotic coronary artery disease. Indeed, consistent with other studies ${ }^{3,7}$, Myasoedova, et al found the risk for heart failure increased in patients with positive rheumatoid factors, persistently elevated sedimentation rates, and the presence of severe extraarticular manifestations of RA. Moreover, one of the key findings in this study is that methotrexate reduced the risk for the development of heart failure, presumably through its control of inflammation. These results are supported by other studies as well ${ }^{8}$. In this context, it is also tempting to speculate that the improved hazard ratio for the development of heart failure in the first year compared to subsequent years can be explained by a delay in controlling inflammation with disease-modifying antirheumatic drugs (DMARD). It may further explain why in this study current but not prior use of these agents affected the development of heart failure.

A beneficial effect on heart failure is not consistent with all antiinflammatory therapies, however. In agreement with prior studies ${ }^{9}$, Myasoedova, et al found that glucocorticoids increased rather than decreased the risk for heart failure. This most likely suggests that glucocorticoids have additional effects that significantly offset their beneficial antiinflammatory effects, perhaps not surprising since glucocorticoids have a host of serious adverse effects such as hypertension, hyperlipidemia, and diabetes, to name a few. It is instructive to note that while current use of glucocorticoids doubled the baseline risk for developing heart failure, methotrexate reduced the baseline risk by half. This provides yet another reason to favor the use of methotrexate over glucocorticoids in the longterm management of RA. These investigators also found that the beneficial and harmful effects of these medications essentially cancelled each other out: in this study there was no change from the baseline risk for heart failure in patients taking both methotrexate and glucocorticoids. This is certainly a fascinating, but at this point, hypothesis-generating observation. While no effect was found in the current study, other work suggests that at least some nonsteroidal antiinflammatory drugs (NSAID) can be detrimental to heart failure ${ }^{8,10}$, providing

See The influence of RA disease characteristics on heart failure, page 1601

Personal non-commercial use only. The Journal of Rheumatology Copyright (C) 2011. All rights reserved. 
an additional rationale to emphasize more aggressive use of DMARD to reduce dependency on these agents as well.

The influence of tumor necrosis factor- $\alpha$ (TNF- $\alpha$ ) inhibitors on heart failure in patients with RA is even more complex. There is evidence that use of TNF- $\alpha$ inhibitors can reduce the risk of RA patients developing atherosclerotic coronary artery disease ${ }^{11}$, thereby also reducing their risk of developing heart failure. The finding of elevated levels of TNF- $\alpha$ in patients with heart failure ${ }^{7,12}$, coupled with animal studies suggesting that TNF- $\alpha$ inhibitors reduced heart failure $^{13}$, led to studies testing whether TNF- $\alpha$ inhibitors would improve heart failure in patients without RA. While the RENEWAL trial found no benefit ${ }^{14}$, patients in the ATTACH study who received TNF- $\alpha$ inhibitors had an increased combined risk for death from any cause or hospitalizations because of worsening heart failure ${ }^{15}$. Because TNF- $\alpha$ inhibitors lower the risk for coronary artery disease $^{11}$, they may nevertheless reduce the risk for developing new-onset heart failure. In observational studies, there is conflicting evidence: one study found that TNF- $\alpha$ inhibitors increased the risk for hospital admissions because of heart failure, even in those without a prior history of heart failure $^{16}$, whereas another study found that TNF- $\alpha$ inhibitors reduced the risk for hospitalizations because of heart failure $^{8}$. It is important to interpret these conflicting data in a wider context. In clinical decision-making, rheumatologists and patients also need to consider the potentially marked benefits TNF- $\alpha$ inhibitors can have on reducing joint destruction as well as improving quality of life.

Clearly, we need more research to improve our understanding of the interrelationships between heart failure and both RA and its treatments. But what do we know now that can improve heart health in patients with RA?

1. Control the traditional risk factors for coronary artery disease in patients with RA. Both primary care physicians and rheumatologists need to increase their vigilance to diagnose and effectively treat these problems to reduce the burden of coronary artery disease and heart failure in their patients.

2. Control inflammation by relying less on NSAID and glucocorticoids and more on DMARD. There is ample evidence that methotrexate, for example, is preferable to either of these medications from a heart point of view. TNF- $\alpha$ inhibitors may be detrimental to patients with uncompensated heart failure and should only be used with caution, if at all, in these patients. Overall, however, the benefits of TNF- $\alpha$ inhibitors likely outweigh their risk in most patients, particularly when we consider the patient as a whole.

3. Increase our vigilance to diagnosis of coronary artery disease and heart failure in patients with RA and not misinterpret their symptoms and signs as manifestations of RA. We can expect better outcomes when we treat the appropriate cause.
Such measures should help us prevent a failure of heart in rheumatoid arthritis.

\author{
MARK L. FRANCIS, MD, \\ Professor of Medicine and Biomedical Sciences, \\ Division of Rheumatology, \\ Texas Tech University Health Sciences Center, \\ El Paso, Texas 79905, USA
}

Address correspondence to Dr.Francis; E-mail: mark.francis@ttuhsc.edu

\section{REFERENCES}

1. Peters MJL, Symmons DPM, McCarey D, Dijkmans BAC, Nicola $\mathrm{P}$, Kvien TK, et al. EULAR evidence-based recommendations for cardiovascular risk management in patients with rheumatoid arthritis and other forms of inflammatory arthritis. Ann Rheum Dis 2010;69:325-31.

2. Myasoedova E, Crowson CS, Nicola PJ, Maradit-Kremers H, Davis JM 3rd, Roger VL, et al. The influence of rheumatoid arthritis disease characteristics on heart failure. J Rheumatol 2011; 38:1601-6.

3. Nicola PJ, Maradit-Kremers H, Roger VL, Jacobsen SJ, Crowson $\mathrm{CS}$, Ballman $\mathrm{KV}$, et al. The risk of congestive heart failure in rheumatoid arthritis. Arthritis Rheum 2005;52:412-21.

4. Francis ML, Varghese JJ, Mathew JM, Koneru S, Scaife SL, Zahnd WE. Outcomes in patients with rheumatoid arthritis and myocardial infarction. Am J Med 2010;123:922-8.

5. Rudominer RL, Roman MJ, Devereux RB, Paget SA, Schwartz JE, Lockshin MD, et al. Independent association of rheumatoid arthritis with increased left ventricular mass but not with reduced ejection fraction. Arthritis Rheum 2009;60:22-9.

6. Crowson CS, Nicola PJ, Kremers HM, O'Fallon WM, Therneau TM, Jacobsen SJ, et al. How much of the increased incidence of heart failure in rheumatoid arthritis is attributable to traditional cardiovascular risk factors and ischemic heart disease? Arthritis Rheum 2005;52:3039-44.

7. Giles JT, Fernandes V, Lima JAC, Bathon JM. Myocardial dysfunction in rheumatoid arthritis: epidemiology and pathogenesis. Arthritis Res Ther 2005;7:195.

8. Bernatsky S, Hudson M, Suissa S. Anti-rheumatic drug use and risk of hospitalization for congestive heart failure in rheumatoid arthritis. Rheumatology 2005;44:677-80.

9. Davis JM 3rd, Kremers HM, Crowson CS, Nicola PJ, Ballman KV, Therneau TM, et al. Glucocorticoids and cardiovascular events in rheumatoid arthritis: a population-based cohort study. Arthritis Rheum 2007;56:820-30.

10. Mamdani M, Juurlink DN, Lee DS, Rochon PA, Kopp A, Naglie G, et al. Cyclo-oxygenase-2 inhibitors versus non-selective non-steroidal anti-inflammatory drugs and congestive heart failure outcomes in elderly patients: a population-based cohort study. Lancet 2004;363:1751-6.

11. Jacobsson LTH, Turesson C, Gulfe A, Kapetanovic MC, Petersson IF, Saxne T, et al. Treatment with tumor necrosis factor blockers is associated with a lower incidence of first cardiovascular events in patients with rheumatoid arthritis. J Rheumatol 2005;32:1213-8.

12. Testa M, Yeh M, Lee P, Fanelli R, Loperfido F, Berman JW, et al. Circulating levels of cytokines and their endogenous modulators in patients with mild to severe congestive heart failure due to coronary artery disease or hypertension. J Am Coll Cardiol 1996;28:964-71.

13. Kadokami T, Frye C, Lemster B, Wagner CL, Feldman AM, McTiernan CF. Anti-tumor necrosis factor- $\alpha$ antibody limits heart failure in a transgenic model. Circulation 2001;104:1094-7.

14. Mann DL, McMurray JJV, Packer M, Swedberg K, Borer JS, 
Colucci WS, et al. Targeted anticytokine therapy in patients with chronic heart failure: results of the randomized etanercept worldwide evaluation (RENEWAL). Circulation 2004;109:1594-602.

15. Chung ES, Packer M, Lo KH, Fasanmade AA, Willerson JT, for the ATTACH investigators. Randomized, double-blind,

placebo-controlled, pilot trial of infliximab, a chimeric monoclonal antibody to tumor necrosis factor-alpha in patients with moderate-to-severe heart failure: results of the anti-TNF therapy against congestive heart failure (ATTACH) trial. Circulation 2003;107:3133-40.

16. Setoguchi S, Schneeweiss S, Avorn J, Katz JN, Weinblatt ME, Levin R, et al. Tumor necrosis factor-alpha antagonist use and heart failure in elderly patients with rheumatoid arthritis. Am Heart J 2008;156:336-41.

J Rheumatol 2011;38:1541-3; doi:10.3899/jrheum.110735 